\title{
Recursos personales y redes de apoyo en el tránsito del joven a la universidad ${ }^{1}$ Personal resources and support networks in the transit of the youth to university
}

\author{
Mireya Ospina Botero ${ }^{2}$ \\ mireya.ospina@ucp.edu.co \\ Stefhany Betancurt Molano ${ }^{3}$ \\ tefa03082@gmail.com \\ María Alejandra Martínez Arias ${ }^{4}$ \\ alejandra.mar.ari@gmail.com
}

\begin{abstract}
Resumen
El presente artículo surge del desarrollo de una investigación de corte cualitativo en relación a las experiencias de un grupo de jóvenes estudiantes de la Universidad Católica de Pereira durante el periodo de adaptación al contexto universitario. La pretensión en este, es el análisis del papel que juegan los recursos personales y las redes de apoyo de los estudiantes en el proceso de transición a la educación superior. En los hallazgos se identifican los recursos personales y sociales de los cuales hacen uso los jóvenes universitarios; se describen las formas en que están consolidadas las redes de apoyo, planteando finalmente los posibles temas de relevancia para la construcción de rutas de acompañamiento e intervención con los estudiantes en la etapa de tránsito.
\end{abstract}

Palabras clave: Educación superior; Proceso adaptativo; Redes de apoyo; Recursos personales.
This article arises from the development of a qualitative research according to the experiences of a group of young students at Universidad Católica de Pereira during the adaptation to the university context period. The purpose of this research is to analyse the role of personal resources and students support networks in the transition process to higher education. Personal and social resources used by higher education students were identified; the ways in which support networks are consolidated are described. Finally, the possible relevant issues for the construction of accompany and intervention routes with the students in the transition stage.

Keywords: Higher education; Adaptive process; Support networks; Personal resources.

Artículo de investigación de la línea en Pedagogía, formación y desarrollo Humano. Universidad Católica de Pereira.

${ }^{2}$ Mg. en Educación y Desarrollo Humano. Docente Universidad Católica de Pereira. ORCID https://orcid.org/0000-0003-4571-8377

${ }^{3}$ Psicóloga. Universidad Católica de Pereira. ORCID https://orcid.org/0000-0001-9376-6045

${ }^{4}$ Psicóloga. Psicóloga clínica IPS Pereira. ORCID https://orcid.org/0000-0002-6609-4041

${ }^{*}$ Cómo citar este artículo: Ospina, M., Betancurt, S. \& Martínez, M. (2018). Recursos personales y redes de apoyo en el tránsito del joven a la universidad. Hojas y Hablas, (15), 100115. DOI: $10.29151 /$ hojasyhablasn.15a6 


\section{Introducción}

La entrada a la educación superior es un momento decisivo para la vida del estudiante, dado que esta consiste en un periodo de transición que surge como un detonante de nuevas adquisiciones y estructuraciones personales con consecuencias tanto en el desarrollo cognitivo como psicosocial (Sousa, Lopes y Ferreira, 2013). Esta transición es un proceso complejo, que comprende el primer año de vida universitaria, el cual implica un conjunto de estrategias para que el estudiante logre readaptarse a una serie de alteraciones significativas, de esta forma, el joven universitario no solo deberá generar nuevas maneras de pensar, sino también nuevas relaciones sociales, es decir, nuevas redes de soporte social. (Aguilera, 2007); (Pérez, et al., 2011).

Dicha transición es una etapa particularmente exigente, donde se registran altos porcentajes de problemas de adaptación, los cuales repercuten directamente en la permanencia de los jóvenes en la institución educativa, preocupando no sólo al estudiante que decide abandonar sus estudios sino también a la institución que no logra su permanencia (Polanco, et al., 2014).

Es por lo anterior que, en las últimas décadas ha cobrado relevancia el proceso de transición puesto que, cada vez es mayor el porcentaje de estudiantes que no logran adaptarse a los requisitos del proceso formativo, por razones como la falta de preparación, información, y de apoyo externo (Álvarez, López y Pérez, 2015; Álvarez, Santiviago, López, Da Re y Rubio, 2014; Herrera, González, Poblete y Carrasco, 2011).

Por consiguiente, Fernández y Polo (2011) sugieren la necesidad de que las universidades provean al estudiante de nuevo ingreso, con una serie de herramientas básicas para que la adaptación al contexto universitario sea lo más exitosa posible. El Ministerio de Educación estipula en los Decretos 2566 del 2003 y 1295 del 2010, que toda institución de educación superior debe asegurar el Bienestar Universitario durante todo el proceso formativo del estudiante.

La mayoría de los estudios acerca del proceso de transición a la educación superior se encuentran enfocados en variables académicas (Álvarez, Figuera y Torrado, 2011; Valdés y Pujol, 2013; Erazo, 2013; Deaño, Alfonso, Conde, Garcia-Señoran, y Tellado, 2014; Barahona, 2014; Álvarez, et al., 2015) y otra parte de estos se dedican a evaluar dimensiones psicosociales, sin embargo, sus descripciones se quedan en un nivel general, dado que no se profundizaban categorías específicas de las características de los estudiantes y se recomienda la elaboración de estudios más específicos que logren analizar el peso particular de cada una de ellas (Álvarez, et al., 2011). Es por esto que en este estudio se tornan relevantes la categoría de recursos personales y la subcategoría de redes de apoyo social como recurso externo del joven estudiante, puesto que ambos hacen parte del componente psicosocial y se presentan como inherentes a la adaptación universitaria.

Inicialmente se encuentra el aspecto de los recursos, los cuales son entendidos como un conjunto de variables que facilitan el desarrollo de la vida del sujeto, estas pueden cambiar y ser potenciadas a través de experiencias previas o por medio del aprendizaje, un recurso constituye un elemento de poder que ayuda a incrementar la calidad de vida y el bienestar subjetivo de las personas. Específicamente, los recursos personales se asocian a los conceptos de capacidades y de fortalezas (Amorós, 2007; Rivera y Andrade, 2006 citado en Rodríguez y Barajas, 2011). Este tipo de recursos se comprenden en cuanto a una dimensión psicológica donde se encuentran rasgos, 
características o habilidades de una persona que pueden ser utilizados para enfrentar un hecho estresante (Ospina y Cardona, 2015).

Estos recursos personales han sido considerados entonces como un arsenal de habilidades con las cuales las personas afrontan diversas situaciones en su vida. Las habilidades para la vida son consideradas como herramientas para el comportamiento adaptativo, que permite a las personas tratar con eficacia las demandas y los desafíos de la vida diaria, estas habilidades son innumerables, y la naturaleza y definición de habilidades para la vida es probable que difieran entre culturas y entornos. Sin embargo, las siguientes habilidades están encaminadas en la promoción de la salud y el bienestar de los adolescentes (OMS, 1997).

La OMS (1997) establece entonces como habilidades para la vida las siguientes: Toma de decisiones, resolución de problemas, pensamiento creativo, pensamiento crítico, comunicativas, relaciones interpersonales, autoconcepto, empatía, control de emociones, control del estrés; sin embargo para el caso de la investigación, de la cual surge este artículo, solo se retoman cinco de estas categorías: el autoconcepto, la toma de decisiones, las habilidades comunicativas, la resolución de problemas y el manejo de emociones debido a su estrecha vinculación con los procesos universitarios.

En relación al autoconcepto se encuentra que es el resultado de la acumulación de autopercepciones obtenidas a partir de las experiencias vividas por el individuo en su interacción con el ambiente, es un concepto que se tiene de sí mismo como un ser físico y social. A medida que pasan los años se va formando un autoconcepto cada vez más estable y con mayor capacidad para dirigir nuestra conducta (Núñez y González, 1994 citado en Luna y Molero, 2013).

En lo referente a la toma de decisiones, como propone Sánchez (2012) se considera como un proceso mental del ser humano, en el que se integran diversas funciones cognitivas e intelectuales, entre las que se encuentran, la memoria en relación a experiencias y aprendizajes, la percepción del estado actual del mundo, la estimación de las consecuencias de cada decisión y la atención puesta a la información relevante. El proceso de tomar decisiones se ha caracterizado entonces como un procedimiento complejo en el cual los individuos identifican y evalúan cursos de acción alternativos y eligen la implementación de uno de ellos (Horan, 1979 citado en Casullo, 1996).

El proceso comunicativo, es concebido como un proceso complejo, de carácter material y espiritual, social e interpersonal que posibilita el intercambio de información, la interacción, e influye directamente en el comportamiento humano, a partir de la capacidad simbólica del hombre. Representa una forma de interacción humana, que está condicionada por el lugar que ocupa el hombre dentro del sistema de las relaciones sociales (Rodríguez, Blanco y Parra, 2009).

La habilidad de resolución de problemas implica la búsqueda racional de soluciones a través de una serie de estrategias que ayudan a afrontar una situación problemática, este es un proceso motivacional que requiere la puesta en marcha de un conjunto de esquemas cognitivoemocionales que reflejan la forma en la que las personas ven los problemas de la vida y su propia habilidad para resolverlos. Estos esquemas, junto con las tendencias conductuales de afrontamiento específicas que les acompañan tienen un efecto facilitador o inhibidor sobre la resolución de problemas (Bados y García, 2014). 
El manejo de emociones supone poseer una serie de habilidades para regular las emociones que permitan a la persona hacerse cargo de la situación, tomar decisiones entre alternativas posibles y reaccionar de manera controlada ante los diversos acontecimientos de la vida. El manejo de emociones puede ser enseñado y aprendido, puesto que persigue encontrar el equilibrio emocional para alcanzar la autonomía y el bienestar personal (Vivas, Gallego y González, 2007).

Vinculado a los recursos personales, se encuentra la búsqueda o el contacto con las redes de apoyo social, las cuales según Sluzki (2002) pueden ser definidas como la suma de todas las relaciones que un individuo percibe como significativas, y estas corresponden al nicho interpersonal de cada sujeto constituyendo una de las claves centrales de la experiencia individual de identidad y bienestar.

La red social es dividida teóricamente en las características estructurales y las funciones de cada atributo, entre las primeras se encuentran el tamaño, el cual hace referencia al número de personas en la red; la densidad, entendida como la conexión entre miembros; la composición o distribución, definida como la proporción del total de miembros de la red y su ubicación; la dispersión, es decir, la distancia geográfica entre los miembros, y finalmente la homogeneidad de los miembros, según edad, cultura y nivel socioeconómico. En cuanto a las segundas, se encuentra, la compañía social, el apoyo emocional, la guía cognitiva y consejos (Sluzki, 2002).

Después de un breve recuento teórico y con base en lo señalado anteriormente en relación a la inherencia de los recursos tanto personales como sociales en los procesos adaptativos, y los vacíos encontrados en la literatura en relación a éste tipo de estudios, surge el interés por cuestionarse respecto al papel que juegan los recursos personales y las redes de apoyo en el proceso de transición a la educación superior en un grupo de jóvenes, específicamente pertenecientes a la Universidad Católica de Pereira.

\section{Metodología}

El presente artículo surge del desarrollo de una investigación de corte cualitativo, que como objetivo general se propuso analizar el papel que juegan los recursos personales y las redes de apoyo, en los estudiantes en el proceso de transición a la educación superior en un grupo de jóvenes de la Universidad Católica de Pereira. Para el cumplimiento de lo anterior, se proponen como objetivos específicos, identificar los recursos del orden personal utilizados por un grupo de jóvenes para adaptarse al contexto universitario, describir las redes en las que participan los jóvenes y la manera en la que estas se configuran como apoyo en el tránsito y finalmente esbozar rutas de intervención y acompañamiento para la institución en función de los procesos de inducción y acompañamiento a los universitarios. Esta investigación se desarrolló durante el periodo comprendido entre junio de 2016 a mayo de 2017.

El grupo de 5 estudiantes participantes tienen edades entre los 17 y 23 años y se encontraban matriculados entre tercer y séptimo semestre en los programas de Comunicación Social y Periodismo, Psicología, Ingeniería Industrial y Arquitectura, de la Universidad Católica de Pereira.

La propuesta metodológica se describe en las siguientes tres fases o momentos:

Identificación de los sujetos participantes

En un primer momento se estableció 
contacto con cada uno de los posibles participantes brindándoles información puntual respecto al estudio a realizar, los beneficios de este para la institución y estudiantes futuros, al igual que para ellos mismos y su proceso de retroalimentación. Finalmente se convoca cada uno al desarrollo de las entrevistas.

\section{Recolección de información}

La recolección de información se desarrolló por medio de los procesos de entrevista, la técnica del mapa de la red propuesto por Sluzki (2002) con el fin de obtener mayor cantidad de información acerca de las redes en las que se apoyan los participantes.

\section{Análisis de hallazgos}

Para el análisis de la información obtenida se desarrollaron matrices categoriales que favorecieron la identificación de categorías emergentes. Ésta es una técnica de análisis que consiste en categorizar y ordenar por atributos de forma reiterativa o repetitiva, cosas, eventos, actos, actores, procesos, escenarios y situaciones dentro de unas categorías determinadas (Quintana y Montgomery, 2006).

\section{Resultados y discusión}

En este apartado se plasman los hallazgos a la luz de los recursos personales y sociales encontrados en la investigación que da surgimiento al presente artículo, estos se presentan sintetizados en dos apartados. En el primero de ellos se identifican los recursos personales y la forma en que los jóvenes se apropian de estos para afrontar la entrada al contexto universitario, y en el segundo, se describen la manera en que están consolidadas las redes de apoyo de cada uno de los universitarios.
Tal como se ha mencionado anteriormente, se parte de la consideración de que el proceso de tránsito a la educación superior es una etapa compleja que requiere del uso de recursos por parte de los jóvenes para el afrontamiento de las transformaciones significativas que se generan en las diversas áreas de los universitarios.

Para efectos de la presentación de las categorías emergentes en los resultados se hará uso de títulos, surgidos de las mismas narrativas de las personas participantes de la investigación y que ilustran su contenido. Dichas narrativas se presentan en cursiva para visibilizar y diferenciar de una mejor manera el hallazgo, en la voz de las personas participantes de la investigación.

\section{“El ingreso a la universidad ha transformado mi forma de ver la vida"}

En este capítulo se van a presentar los recursos de orden personal identificados en los estudiantes que les posibilitaron hacer un tránsito, para ello se describen los hallazgos alrededor de los siguientes recursos: autoconcepto, toma de decisiones, resolución de problemas, habilidades comunicativas y manejo de emociones

\section{Autoconcepto}

El tránsito a la educación superior se presenta como una oportunidad para la adquisición de nuevos conocimientos tanto a nivel académico, como personal y social, los cuales posibilitan nuevas formas de comportarse frente al mundo, dado que los estudiantes empiezan a percibirse a sí mismos y a los demás de una manera diferente, una más responsable y crítica tal como lo plantea uno de los participantes:

"He adquirido mayor carácter, mayor compromiso respecto a la universidad y a otras 
actividades que realizo, siento como que he madurado...".

Estas nuevas formas de comportarse frente al mundo, generadas por la adquisición de nuevos conocimientos, se entendería entonces como el cambio obtenido a partir de experiencias vividas, en la forma de percibirse y de actuar del sujeto, así como plantea Núñez y González (1994) citado en Luna y Molero, (2013), a medida que pasan los años, en este caso a medida que transcurre la entrada a la educación superior, se va formando un autoconcepto cada vez más estable y con mayor capacidad para dirigir la conducta, aspecto que facilitaría la identificación por parte del sujeto de fortalezas y debilidades que posibilitarían la puesta en marcha de recursos adecuados para la adaptación a la vida universitaria.

Los estudiantes se valen de recursos identificados previamente a la entrada a la universidad, para el afrontamiento de esta nueva etapa adaptativa, una de estas fortalezas que comparten algunos de participantes son las habilidades sociales, como se logra evidenciar en el siguiente fragmento:

"Una fortaleza que poseo es que soy muy sociable, pienso que esa cualidad fue una de las que más me ayudó porque apenas entre a la u pude crear lazos muy fuertes, entonces siempre tengo algún apoyo."

El ingreso a la universidad supone un ajuste en las expectativas de los estudiantes, puesto que al encontrarse con la realidad que les brinda este nuevo contexto, ellos empiezan a identificar la relevancia de estas expectativas previas y es entonces cuando debe hacer uso de la nueva información para replantearse sus metas y la forma de llegar a ellas, como lo expresa el siguiente participante:
"Al inicio uno entra con un propósito, pero en la medida en la que se avanza en los estudios, las perspectivas cambian y los objetivos se ven modificados, y ya en este punto de mi carrera, pienso que tengo otros enfoques otras motivaciones".

Los estudiantes en esa etapa de la vida se encuentran en la búsqueda y el establecimiento de lo quieren ser como personas, definiendo metas, objetivos, motivaciones, formas de ver el mundo, en esta medida la universidad se presenta como una entidad que brinda información sociocultural, conocimientos académicos y toda una serie de experiencias individuales y sociales, que van a posibilitar el establecimiento de sus formas de ser y actuar frente a la interacción con el mundo interno y externo, como lo manifiesta uno de los participantes a continuación:

"El ingreso a la universidad hasta este momento me ha hecho modificar muchas pautas en mi comportamiento, en la manera de pensar, sentir, porque anteriormente era una persona que no veía hacia delante...".

Lo anterior concuerda con lo establecido por Clark, Clemes y Bean (2000) citado en Luna y Molero, (2013) donde esbozan la importancia de la función del autoconcepto como pilar en la formación de la personalidad, ya que este influye sobre la persona en cómo se siente, cómo piensa, cómo aprende, cómo se valora, cómo se relaciona consigo mismo y con los demás y, en definitiva, cómo se comporta.

La universidad se presenta como un contexto lleno de nuevos retos para el estudiante, tal como lo plantea Pérez, et al. (2011) al esbozar que la adaptación al contexto universitario es un proceso complejo, multifactorial, que implica un conjunto de estrategias para que el estudiante 
logre readaptarse a una variada serie de alteraciones significativas. Al momento de afrontar estos retos, las creencias que tienen frente a sí mismos, y las capacidades con las que creen contar para responder frente a estas, van a jugar un papel determinante en la forma en la que afronten la situación y los sentimientos que les generen, puesto que entre mayor confianza tengan los estudiantes en sí mismo, tomarán las dificultades como retos y no como obstáculos que retrasen su proceso. Esto logra ser evidente en la siguiente narrativa:

"Si no hubiera sido por la confianza que me tengo entonces no estuviera en este nivel, porque ya es tercer semestre $y$ he considerado que el proceso no es fácil"

La importancia de la autopercepción de capacidades y habilidades que se menciona en el párrafo anterior, es apoyada por Goñi (2009), al mencionar que cuando se cuenta con un autoconcepto positivo se compone la base del buen funcionamiento personal, social y profesional, dado que, de él depende en gran medida la satisfacción personal.

\section{Toma de decisiones}

La educación superior supondría una influencia directa en el aspecto académico de las personas que ingresan, sin embargo la entrada al contexto universitario logra permear cada ámbito de la vida del ser humano, es decir, se ven movilizados el aspecto social, personal, familiar, económico, profesional, espiritual y comunitario, este aspecto multidimensional del contexto universitario se evidencia en los discursos de los participantes, dado que al abordar por las decisiones más importantes que han debido tomar, ellos mencionan decisiones en relación a todas las anteriores esferas.
La entrada a la universidad suele suponer el fortalecimiento de la autonomía, puesto que, las instituciones de primaria y secundaria tienden a brindar un acompañamiento permanente a los jóvenes, por el contrario, uno de los propósitos fundamentales de la educación superior es potenciar los mecanismos de independencia y autonomía, es por esto que exige el planteamiento de un proyecto de vida, proyecto que demanda una posición más crítica frente a los demás, el mundo, y sí mismo, teniendo como fin una toma de decisiones asertivas y congruentes frente a sus metas y proyectos, idea que se esboza en el siguiente discurso:

"Aprende uno a ser más crítico, a escuchar más a las personas, a entender más, entonces las decisiones las tomo como mirando otro punto de vista, de esta manera he aprendido como a hacer algunos ejercicios como para el proceso de tomar decisiones."

Esta importancia de toma de decisiones asertivas, es respaldada por Biel, Soldevilla, Arbej y Fombuena (2000) quienes aseguran que, mediante este estilo cognitivo, las personas tienen la oportunidad de aprender a tomar decisiones, dado que el manejar constructivamente las decisiones tendrá consecuencias positivas en la vida de las personas, especialmente en los estudiantes que se encuentran en la etapa universitaria, dado a la gran cantidad de cambios y la constante necesidad de dar respuesta a estos de la forma más asertiva posible.

\section{Habilidades comunicativas}

Al momento de enfrentar nuevas experiencias, encontrar nuevas personas, nuevas formas de pensar, sentir y actuar, como lo implica la entrada a la universidad, las habilidades comunicativas se presentan como un recurso útil y efectivo 
que contribuyen en los procesos de interacción generando recursos para la adaptación universitaria, tanto a nivel social como académico, en lo social con el mejoramiento de los mecanismos de interacción, tal y como lo expresa uno de los participantes:

"Es muy importante porque realmente en la entrada a la universidad uno lo primero que debe hacer es buscar un soporte, alguien con quien estar, entonces yo pienso que las habilidades comunicativas deben ser bastante amplias para que uno no se sienta solo".

Tal como lo esbozan los autores Viana y Lima (2011), refiriendo que las habilidades comunicativas son el medio que permite establecer relaciones interpersonales, conservar e interactuar con otras personas de forma positiva. Para facilitar la comunicación, es necesario tener una comunicación asertiva, entendida como la destreza que nos permite expresar sentimientos, opiniones y pensamientos, en el momento pertinente, de la forma adecuada sin ofender ni causar ningún problema con el entorno.

En el aspecto académico, la comunicación asertiva le permite al universitario una visión de posibles compañeros de trabajo, la identificación de un grupo afín con sus características individuales, buen desempeño en actividades expositivas, y una adecuada expresión de ideas a los demás, como lo refiere el participante:

"Creo que eso es bastante importante en la forma en la que uno debe desempeñarse tanto académicamente como laboralmente y no solo dentro de la carrera sino también en otros contextos donde es importante comunicarse y saber comunicar".
Rodríguez, et. al. (2009) respaldan la idea anterior, definiendo el proceso comunicativo como un proceso complejo, de carácter material y espiritual, social e interpersonal que posibilita el intercambio de información, la interacción, e influye directamente en el comportamiento humano, a partir de la capacidad simbólica del hombre. Representa una forma de interacción humana en diferentes contextos.

La importancia de las habilidades comunicativas en ambos aspectos, sociales y académicas, se perciben de forma clara en el relato del siguiente participante:

"Yo tuve la oportunidad de relacionarme con personas y pues comunicándome con ellos es que he podido solucionar cosas, he tenido ayuda por parte de ellos, y si no fuera por eso estaría solo y no tuviera quien me ayudara. Los profesores lo notan a uno, y por cosas de esas uno salva materias".

Es evidente entonces, que el contexto universitario es transversalizado por el uso de las habilidades comunicativas, dado que son estas las que posibilitan el establecimiento de relaciones interpersonales y el acceso a información contextual por parte del sujeto transitante, dado que, como plantea Álvarez, et al. (2015) el joven universitario al ingresar requiere de forma inmediata informarse respecto al nuevo contexto, las nuevas dinámicas, y formas de involucrarse e interactuar en las mismas; y es allí cuando las habilidades comunicativas posibilitan la interacción con diferentes actores universitarios, que pueden ser sus propios compañeros estudiantes, docentes, administrativos o personas que laboren en la institución. 
Resolución de problemas

Son diversas las dificultades o situaciones problemáticas que se presentan en la entrada al contexto universitario, en el grupo de estudiantes entrevistados se identifica en cada uno la capacidad activa de resolución de conflictos, dado que, encontraron formas de afrontar estos obstáculos de manera satisfactoria, la situación problemática encontrada en el grupo de universitarios fue la dificultad de accesibilidad a recursos materiales, aspecto que se logra vislumbrar en la siguiente narrativa:

"Yo tuve dificultades con los pasajes, los almuerzos, pero yo empecé a vender dulces y por medio de eso me ayudaba con esos gastos, ya después desde el semestre pasado empecé a trabajar".

Vale la pena decir que todos los seres humanos están expuestos a una serie de vivencias que demandan en ellos la capacidad de afrontar y resolver situaciones que son significadas como conflicto o problema, como el aspecto económico en los jóvenes universitarios, y es allí cuando se torna relevante lo plateado por Guerra (s.a), al expresar la importancia del entrenamiento en la resolución de problemas, dado que este proceso cognitivo y comportamental ayudaría al sujeto a hacer disponibles una variedad de alternativas de respuesta lo que contribuye a incrementar la probabilidad de seleccionar las opciones más eficaces de entre las alternativas posibles y solucionar dicha situación problemática.

\section{Manejo de emociones}

La entrada al contexto universitario supone la presencia de un sin número de emociones, dado la complejidad del proceso y la variedad de experiencias que afectan lo que cada sujeto ha construido respecto a sí mismo, emociones que en su mayoría generan inestabilidad. Frente a este malestar emocional los jóvenes desde sus saberes y experiencias previas, haciendo uso de sus recursos, le hicieron frente a este malestar, logrando canalizar sus emociones en diferentes actividades, unas brindadas por el ambiente universitario y otras propuestas por los mismos estudiantes. En esta búsqueda de estrategias, los jóvenes se apoyaron en sus redes familiares y sociales, algunas previamente establecidas y otras que surgieron con la entrada a la universidad, viéndose entonces la estrecha relación entre el recurso interno de manejo de emociones y el recurso externo de acompañamiento por parte de las redes de apoyo social; en el caso de redes antiguas, una de las participantes expresa:

"Cuando me sentía mal me ponía a llorar y llamaba a mi novio, lo llamaba a decirle cómo me sentía y él me ayudaba un poquito".

Y frente a las redes en proceso de consolidación:

"Me apoyaba en los otros compañeros que apenas estaba conociendo, nos acompañamos en el sentimiento de los nervios".

Como se menciona en la idea anterior, una de las estrategias encontradas por los jóvenes universitarios para hacerle frente a su malestar emocional fueron las redes de apoyo, familiares o sociales, es aquí donde puede empezar a vislumbrarse la relación existente entre las categorías de análisis del actual estudio, las redes de apoyo son consideradas entonces como otro de los tipos de recursos de los que hacen uso los jóvenes en su tránsito a la educación superior, lo relacionado a las redes es abordado en el siguiente capítulo. 
De tal manera que, el tránsito a la educación superior se presenta como un proceso complejo que implica en los jóvenes universitarios la puesta en marcha de una amplia gama de recursos de tipo personal, material y social para lograr responder de manera satisfactoria a este nuevo contexto.

\section{"Mi red de apoyo, las personas con las que real- mente puedo contar"}

En este apartado, se realiza una breve presentación de los recursos de tipo social, es decir, las redes de apoyo, describiendo la forma en que estas redes se constituyen y se configuran como un apoyo para el joven frente al tránsito a la universidad, por lo tanto, la presentación de este recurso se desarrolla en referencia a las características estructurales y las funciones de cada atributo, tal como lo propone Sluzki (2002). Entre las primeras se encuentran el tamaño, la densidad, la composición o distribución, la dispersión, y la homogeneidad, y en cuanto a las segundas se encuentra, la compañía social, el apoyo emocional, y la guía cognitiva o consejos.

De ahí que, el medio por el cual se facilitó la exploración de las redes de los jóvenes universitarios, fue el "Mapa de la red"; este mapa consiste en un entramado mínimo sistematizado en cuatro cuadrantes o áreas sociales (Familia, Amistades, Relaciones laborales o escolares, Relaciones comunitarias), sobre los cuales se inscriben tres delimitaciones en referencia a la proximidad con el sujeto, uno próximo a él, uno intermedio, y otro externo (Sluzki, 2002).

Frente a estos resultados, no es labor de los investigadores o los profesionales definir lo insuficiente o suficiente de una red, son las personas, en este caso los participantes quienes expresan su grado de satisfacción frente al tamaño de sus redes, aunque algunas de las personas sienten que el número de personas que conforman su red no es suficiente, todos los participantes concuerdan en sentirse satisfechos con las personas que hacen parte de esta, lo cual se evidencia en el relato del siguiente participante:

"No son muchas personas, pero están las más allegadas a mí, las personas más importantes, con las cuales puedo contar y me apoyo".

Las redes de los participantes en cuanto a tamaño se encuentran un número promedio de 15 personas.

En las redes de los participantes se encuentran diversos grupos definidos, la conexión entre estos grupos logra evidenciarse como alta, lo que permite que los sujetos perciban la red como más eficaz y les permite obtener recursos para afrontar el proceso de adaptación a la educación superior. Entre los grupos que se evidencian como más unidos, encontramos a la familia, y los amigos íntimos, como se logra percibir en lo expresado por el siguiente participante:

"Por parte de mi familia pues todos son muy cercanos, todos son muy unidos y ellos conocen a mis amigos".

Esto es lo denominado por Sluzki (2002) como densidad, los subsistemas entre la red y su capacidad de influencia de estos en el individuo, es decir la relación de los grupos con el individuo.

La familia es planteada por los participantes como el punto de conexión entre los demás cuadrantes de la red, puesto que es este grupo quien informaría a los demás miembros sobre alguna situación problemática, es decir la familia es 
percibida como el punto de accesibilidad para los demás miembros de la red, aspecto que logra vislumbrarse en el siguiente discurso:

"Mis papás se encargarían de regar la información. Ellos son la parte principal para decirles a todos".

En palabras de Sluzki (2002), la distancia geográfica entre los miembros, entendida como accesibilidad, es lo que facilitaría el acceso o contacto para generar comportamientos efectivos, $y$ lo que se pudo observar en los participantes es que la familia representa ese punto de acceso entre el sujeto y el resto de la red.

Respecto a lo que Sluzki (2002) define como composición o distribución, las redes realizadas por los participantes se presentan como distribuidas, es decir, se encuentran miembros en cada cuadrante, y tienden a encontrarse en el círculo íntimo, sin embargo, en algunas de las redes se evidencia una ausencia en el cuadrante de grupos institucionales $y$, por el contrario, se identifica una gran cantidad en los cuadrantes de familia y amigos, aspecto que logra evidenciar el siguiente participante:

"En mi familia se encuentran más personas, pero en mis amigos también hay como varios".

Respecto a la homogeneidad de las redes (Sluzki, 2002) se encuentra gran diversidad en cuanto a los tipos de miembros pertenecientes a los cuadrantes, diversidad en cuanto a niveles educativos, edades, profesiones, relaciones afectivas, lo cual denominaría a las redes desarrolladas como heterogéneas, lo que permite riqueza en la red y apoyo más efectivo en cuanto a cubrimiento de diversos campos.
En relación al acompañamiento socioafectivo que generan las redes de apoyo en los sujetos, coincide lo desarrollado por Sluzki (2002) frente a que el apoyo y compañía emocional es una función característica de las amistades intimas y las relaciones familiares, con lo expuesto por los participantes en sus redes, dado que las personas con las que más realizan actividades, se sienten comprendidos y en quienes pueden confiar son la familia y sus mejores amigos, como se observa en los siguientes relatos:

"Por quien me siento comprendida y puedo confiarle mis cosas es a Sara mi mejor amiga y a mi mamá".

"Siempre he compartido la mayoría de tiempo con mi mamá, y pues en este momento también con Sebastián que es como mi mejor amigo".

De igual forma, en lo referente a guías cognitivas u orientación (Sluzki, 2002), es decir interacciones destinadas a compartir información personal o social, se encuentra en la mayoría de redes que los miembros destinados a brindar este tipo de ayudas son los compañeros de universidad dado que este es el contexto donde se desenvuelven los sujetos actualmente, y es allí de donde requieren más información y orientación, como se identifica en el siguiente discurso de uno de los participantes:

"Que me ayuden con cosa, los de la universidad porque son los que me acompañan en mi proceso de estudio entonces es más fácil entenderles a ellos, ellos saben más de eso"

En síntesis, las redes son percibidas como una de las más potentes herramientas a la hora de afrontar las situaciones en relación al contexto universitario, puesto que, en esta etapa, particularmente exigente, la efectividad de estos apoyos 
es percibida en su forma más clara. Estas redes no solo posibilitan la expresión emocional de los jóvenes universitarios, sino que éstas también brindan conocimiento en relación a aspectos académicos o monetarios.

\section{Conclusiones}

Durante el desarrollo del artículo se ha expuesto la complejidad de este proceso adaptativo, y la importancia de contar con recursos de orden personal, social y material para el afrontamiento adecuado del mismo.

En el orden de los recursos personales, logra identificarse en los discursos de los participantes, la primacía de las denominadas habilidades para la vida, y su rol fundamental en los procesos cotidianos, los sujetos investigados hacen referencia a la importancia de los procesos de interacción (habilidades comunicativas), la transformación que se genera en sus formas de pensar y actuar debido a la entrada al contexto universitario (autoconcepto), las decisiones a las que debieron enfrentarse en relación a sus proyectos académicos (toma de decisiones), las dificultades que fueron surgiendo mediante el proceso y las formas en que lograron superarlas (resolución de problemas), y finalmente la búsqueda activa de herramientas tanto externas como internas para canalizar sus emociones (manejo de emociones).

El proceso de adaptación al contexto universitario no solo implica al sujeto que transita, sino también a la propia institución en la que este se encuentre, dado que dicha transición es una etapa particularmente exigente donde se registran altos porcentajes de problemas de adaptación y de bajo rendimiento académico, los cuales repercuten directamente en la permanencia de los jóvenes en la institución educativa, aspecto que preocupa entonces a la institución que no logra su permanencia (Álvarez, et. al 2011; Torrado, et. al. (2013); Polanco, et. al. 2014). De igual forma, en los Decretos 2566 y 1295, se explicita la función social de las instituciones de educación superior en relación a asegurar el bienestar universitario desde el ingreso de los estudiantes hasta el momento de finalizar su carrera.

Es por lo anterior, que se torna relevante para las instituciones de educación superior la puesta en marcha de rutas o lineamientos de acompañamiento para la prevención del ausentismo ligado a inadecuados procesos adaptativos, y la promoción del bienestar universitario.

Como se ha planteado desde el inicio de la investigación, el estudiante que ingresa a la educación superior requiere de forma prioritaria informarse respecto a la nueva cultura, las nuevas dinámicas que en este espacio se desarrollan, y formas de involucrarse e interactuar con las mismas; una de las estrategias más directas de llegar a este conocimiento es por medio de la comunicación con diferentes actores universitarios, sean otros estudiantes, docentes, administrativos o personas que laboren en la institución (Álvarez et. Al, 2015).

En ese sentido y en relación a los recursos personales, las habilidades comunicativas se presentan como un recurso útil y efectivo que contribuyen en los procesos de interacción, generando nuevos recursos al momento de enfrentar nuevas experiencias, encontrar nuevas personas, nuevas formas de pensar y sentir, por ende es de vital importancia que las universidades se encaminen en fortalecer y promover las habilidades comunicativas en los universitarios desde el momento 
en que estos ingresen a la institución, ofreciendo espacios que permitan la identificación de estilos, fortalezas y debilidades en torno a los procesos comunicativos y brindando herramientas para la potencialización de los mismos.

Por otro lado, la adaptación al contexto universitario es un proceso complejo, como lo refieren Pérez y cols. (2011), lo que implica un conjunto de estrategias para que el estudiante logre readaptarse a una variada serie de transformaciones significativas, al momento de afrontar estas experiencias, las creencias que tienen frente a sí mismos, y las capacidades con las que creen contar para responder frente a estas, es decir, la autoeficacia, va a jugar un papel determinante en la forma en la que los jóvenes afronten la situación y los sentimientos que les generen.

Por ende, es necesario la implementación de estrategias que contribuyan al afianzamiento de la percepción de autoeficacia del joven universitario, permitiéndole el reconocimiento y la aplicación de aquellos pensamientos referidos a sus capacidades y habilidades para plantearse determinados logros y actuar en consecuencia de esto. Este aspecto se vincula directamente como la habilidad referida al autoconcepto, dado que en él se incluyen acciones encaminadas al reconocimiento, la aceptación y potencialización de características puntuales de cada uno de los jóvenes universitarios.

Por otra parte, esta etapa de transición se muestra como un momento óptimo para comenzar a cristalizar proyectos que llevarán a los estudiantes a ejercer nuevos roles, establecer nuevas decisiones, crear nuevos vínculos afectivos y transformar sus expectativas teniendo presente sus metas a futuro, de igual forma, se requiere de constantes ajustes que suponen afrontar de manera positiva los problemas cotidianos $y$, las dificultades emocionales inherentes al dinamismo de la adaptación universitaria (Sandoval, 2012). Es entonces esta etapa un conjunto de situaciones vitales por las que atraviesa el universitario que requieren, como se ha expuesto anteriormente una amplia gama de recursos, tanto personales como sociales, y en este caso la construcción de un proyecto de vida (Montauti, Bory y Tricio, 2011).

La construcción del futuro personal o proyecto de vida desde el inicio del proceso universitario abarca todas las esferas del ser humano, y requiere de la elaboración de distintas estrategias, actividades, comportamientos y pautas que el sujeto seguirá a lo largo de la carrera universitaria a fin de poder alcanzar las metas planteadas en un inicio, es por esto que el proyecto de vida se constituye como la base para el funcionamiento de las diversas actividades desarrolladas por el estudiante, lo cual debe encontrarse en concordancia con sus objetivos trazados y un adecuado proceso adaptativo.

Por lo anterior, la incidencia que tiene la construcción de un proyecto de vida personal en el tránsito a la universidad, se presenta como una estrategia de prevención para establecer pautas dirigidas a la identificación, definición y fortalecimiento de objetivos a mediano y largo plazo.

Anteriormente se hace mención de la utilidad y necesidad de los recursos de tipo personal en el tránsito, sin embargo, en el presente artículo también es relevante la puesta en marcha de recursos del orden social, como lo son las redes de apoyo de cada sujeto.

Las redes de los participantes se caracterizan en su mayoría por constituirse como redes de tamaño reducidas, percibidas como eficaces puesto que generan en el joven un anclaje entre 
su contexto universitario y su contexto familiar; de igual forma la conexión entre los grupos presentes en cada red es alta, lo que permite que los sujetos perciban con mayor facilidad la eficacia de sus redes, encontrándose que la familia es percibida como el punto de conexión y accesibilidad para los demás miembros de la red; así mismo, en las redes se encuentra gran diversidad en cuanto a los tipos de miembros, niveles educativos, edades, profesiones, y relaciones afectivas, heterogeneidad que permite la riqueza en cada red. Finalmente, en relación al acompañamiento socioafectivo que generan dichas redes esta se concibe como una función característica de las amistades intimas y las relaciones familiares.

Es por lo anterior que, se evidencia la importancia de la vinculación de las redes de apoyo establecidas por el estudiante a los procesos universitarios, dado el rol de acompañamiento y soporte que desempeñan éstas en el proceso de adaptación universitaria, en medida de esto las instituciones de educación superior, podrían plantearse la realización de actividades lúdicas y formativas en compañía de las redes de apoyo de los jóvenes.

Finalmente, después de lograr identificar la amplia gama de recursos tanto personales como sociales que ponen en marcha los jóvenes universitarios para el tránsito, se reconoce entonces que la falta de esta diversidad de recursos, puede presentarse en los universitarios como un factor de riesgo, es decir, si un joven cuenta con mínimas habilidades comunicativas, un bajo reconocimiento de su autoconcepto, inadecuadas estrategias para tomar decisiones, resolver problemas y manejar emociones, y unas redes de apoyo poco consolidadas o escasas, esto influirá directamente en el acceso a la información, el proceso de interacción, la elección de metas a futuro, el afrontamiento de conflictos y la consolidación de nuevas redes, lo cual afectará y de cierta forma enlentecerá el proceso de adaptación al contexto universitario, posibilitando entonces la llegada al ausentismo estudiantil.

\section{Referencias}

Aguilera, M.C. (2007). La transición a la vida universitaria. Éxito, Fracaso, Cambio y Abandono. Comunicación presentada en IV Encuentro Nacional de Docentes Universitarios Católicos.

Álvarez, G. M., Figuera G. P., y Torrado F. M. (2011). La problemática de la transición Bachillerato-Universidad en la Universidad de Barcelona. Revista Española de Orientación y Psicopedagogía, 15-27.

Álvarez, P. P. R., López A. D., y Pérez J. D. (2015). Análisis de los factores que intervienen en la transición del bachillerato a la Universidad. In Investigar con y para la sociedad. 599608.

Álvarez, P. P. R., Santiviago, C., López A. D., Da Re, L., y Rubio, V. (2014). Competencias De Adaptabilidad y Expectativas del Alumnado en Proceso de Transición a la Educación Superior: Un Estudio Transnacional en España, Uruguay E Italia. Cuarta Conferencia Latinoamericana Sobre el Abandono en la Educación Superior.

Amorós, G. M. (2007). Evaluación de un programa de potenciación de los recursos psicológicos para la mejora del bienestar psicológico y de la salud. Madrid: Universidad Autónoma de Madrid

Bados, A., y García, E. (2014). Resolución de problemas. Universitat de Barcelona.

Barahona, P. (2014). Factores determinantes del rendimiento académico de los estudiantes de la Universidad de Atacama. Estudios pedagógicos. 40(1), 25-39.

Biel, C; Soldevilla, A; Arbej, J y Fombuena, M. (2000). Desarrollo de habilidades sociales para el trabajo en promoción de la salud. Trabajo social y Salud. 35. 
Casullo, M. M. (1996). El proceso de tomar decisiones. En M. M. Casullo, \& A. N. Cayssials, Proyecto de Vida y Decisión Vocacional. 31 - 64. Buenos Aires: Paidos.

Deaño, M. D., Alfonso, S. A., Conde, A., GarciaSeñoran, M., \& Tellado, F. (2014). Expectativas universitarias y predicción del rendimiento académico. Revista E-Psi. 4(1), 127-141.

Erazo, S. O. A. (2013). Caracterización psicológica del estudiante y su rendimiento académico. Revista Colombiana de Ciencias Sociales, 4(1), 23-41.

Fernández, J, C., y Polo S. M. T. (2011). Afrontamiento, estrés y bienestar psicológico en estudiantes de Educación Social de nuevo ingreso. EduPsykhé: Revista de psicología y psicopedagogía, 10(2), 177-192.

Goñi, P. E. (2009). El autoconcepto personal: estructura interna, medida y variabilidad. Servicio Editorial de la Universidad del País Vasco/EuskalHerrikoUnibertsitatearenArgitalpenZerbitzua.

Guerra, V. G. (S.A) La Solución de Problemas. Academia de Ciencias de Nueva York.

Herrera, R. H., González, E., Poblete, Á., \& Carrasco, S. (2011). Transición entre educación media y universidad: Marco de referencia y experiencias internacionales. El Proceso De Transición Entre Educación Media Y Superior. Experiencias Universitarias, 51-59.

Luna, N. C., y Molero, D. (2013). Revisión teórica sobre el autoconcepto y su importancia en la adolescencia. Revista Electrónica de Investigación y Docencia (REID), (10).

Ministerio de Educación Nacional. (2003). República de Colombia.

Montauti, E. E., Bory, G. S., y Tricio, E. S. (2011). La Construcción De Los Proyectos De Vida En Jóvenes Escolarizados De Los Partidos De Influencia De La Untref: Un Estudio De Sus Trayectorias. En III Congreso Internacional de Investigación y Práctica Profesional en Psicología XVIII Jornadas de Investigación Séptimo Encuentro de Investigadores en Psicología del MERCOSUR. Facultad de Psicología-Universidad de Buenos Aires.

OMS (1997). Life Skills Education for Children and Adolescents in Schools. Genova.

Ospina B. M., y Cardona G.Y. P. (2015). Un escalón más... la llegada de la familia a la universidad. Pereira: Grafias.

Pérez, C., Silva, E., Jiménez, G., Cáceres, G., Lagos, J., Loncomilla, L., Bagnara, M., Báez, M., Abarca, M. y Ampuero, N. (2011). Análisis y sistematización de experiencias de transición realizadas en Universidades del grupo Operativo Coordinado por Cinda. El Proceso De Transición Entre Educación Media Y Superior. Experiencias Universitarias, 131175.

Polanco, A., Ortiz, L., Pérez, C., Parra, P., Fasce, E., Matus, O., Torres, G. y Meyer, A. (2014). Relación de antecedentes académicos y expectativas iniciales con el bienestar académico de alumnos de primer año de medicina. FEM: Revista de la Fundación Educación Médica, 17(4), 205-211.

Quintana, A., \& Montgomery, W. (2006). Psicología: Tópicos de actualidad. Universidad Nacional Mayor de San Marcos. Facultad de Psicología.

Rodríguez, C. T. L., Blanco A. M. Á., y Parra V. I. B. (2009). Las habilidades comunicativas en la entrevista médica. Revista Cubana de Medicina Militar, 38(3-4).

Rodríguez, M. M., y Barajas, D. D. (2011). El impacto del rol ocupacional de la madre en el desarrollo de recursos psicológicos en adolescentes tardíos. (memorias) México: Congreso Nacional de investigación educativa.

Sánchez, Á. I. (2012). Modelo computacional cognitivo de toma de decisiones basado en el conocimiento: aplicación en la inferencia de explicaciones. (Tesis pregrado) Universidad Complutense de Madrid.

Sandoval, P. L. (2012). Cambios en los referentes para la construcción de proyectos de vida juvenil. Tendencias \& Retos, 17(1), 93-103. 
Sluzki, C. E. (2002). La Red Social: Frontera de la Práctica Sistémica. Barcelona: Gedisa.

Sousa, R., Lopes, A., \& Ferreira, E. (2013). La transición y el proceso de adaptación en la Educación Superior: un estudio con estudiantes de una escuela de enfermería y de una escuela de educación. REDU. Revista de Docencia Universitaria, 11(3), 403-422.

Valdés, M. A., y Pujol, L. (2013). Autorregulación y rendimiento académico en estudiantes universitarios de reciente ingreso: un estudio longitudinal. Educación y futuro. (29), 205-219
Viana, O. Y. A., y Lima A. L. (2011). Proyecto de habilidades para la vida en niños y adolescentes de la asociación cristiana de jóvenes zona San Cristóbal sur: prepárate para la vida, alimenta tu cuerpo y tu mente. Departamento de Nutrición Humana, Facultad de Medicina, Universidad Nacional de Colombia

Vivas, M., Gallego, D., y González, B. (2007). Educar las emociones. Venezuela: Editoriales C. A.

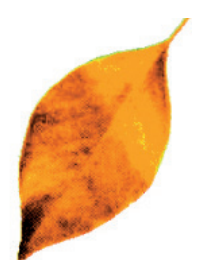

